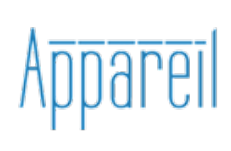

Appareil

1 | 2008

Le milieu des appareils

\title{
Ce que « medium » peut vouloir dire : l'exemple de la photographie
}

\section{Jacques Rancière}

\section{(2) OpenEdition}

\section{Journals}

Édition électronique

URL : http://journals.openedition.org/appareil/135

DOI : 10.4000/appareil. 735

ISSN : 2101-0714

Éditeur

MSH Paris Nord

Référence électronique

Jacques Rancière, «Ce que «medium » peut vouloir dire : l'exemple de la photographie », Appareil [En ligne], 1 | 2008, mis en ligne le 17 février 2008, consulté le 30 juillet 2020. URL : http:// journals.openedition.org/appareil/135; DOI : https://doi.org/10.4000/appareil.135

Ce document a été généré automatiquement le 30 juillet 2020

\section{(c) (i) (9)}

Appareil est mis à disposition selon les termes de la Licence Creative Commons Attribution - Pas d'Utilisation Commerciale - Pas de Modification 4.0 International. 


\title{
Ce que « medium » peut vouloir dire : l'exemple de la photographie
}

\author{
Jacques Rancière
}

1 Je présenterai ici quelques remarques sur l'usage de la notion de médium dans la pensée de l'art et sur l'éclairage qu'apporte à cette notion le cas de la photographie. La notion de médium est en effet beaucoup plus complexe qu'il n'y paraît d'abord. La théorisation du médium comme élément crucial du modernisme artistique fait jouer deux sens apparemment opposés du mot. Dans le mot «médium », on entend d'abord «ce qui se tient entre»: entre une idée et sa réalisation, entre une chose et sa reproduction. Le médium apparaît ainsi comme une intermédiaire, comme le moyen d'une fin ou l'agent d'une opération. Or la théorisation moderniste qui fait de la «fidélité au médium» le principe de l'art renverse la perspective. Ce médium à la spécificité duquel il faut être fidèle n'est plus simplement l'instrument de l'art. Il devient la matérialité propre qui définit son essence. C'est bien le cas dans la définition greenbergienne de la peinture fidèle à son médium propre- la surface bidimensionnelle et le pigment coloré-, et délivrée par là des tâches serviles de la représentation. Le médium n'est plus alors le moyen d'une fin. Il est proprement ce qui prescrit cette fin. Mais la thèse qui identifie l'essence d'un art à la loi de son médium se laisse lire en deux sens opposés. D'un côté, elle dit : l'art est de l'art quand il est délivré des tâches de la mimesis, quand il devient seulement l'exécution dans son matériau propre de sa propre idée. C'est cet énoncé qu'on retient d'ordinaire. Mais la thèse se lit aussi à l'envers : l'art est de l'art quand la contrainte du matériau et de l'instrument le délivre de lui-même, le délivre de la volonté de faire de l'art. La séparation de l'art avec la mimésis est alors aussi une séparation de la tekhnè avec elle-même : séparation entre la tekhnè comme exécution d'une idée, mise en œuvre d'un savoir, et la tekhnè comme loi de la matière et de l'instrument, loi de ce qui n'est pas de l'art.

2 La thèse du médium dit ainsi deux choses à la fois : premièrement l'art est de l'art quand il n'est que de l'art; deuxièmement l'art est de l'art quand il n'est pas que de l'art. On peut synthétiser les deux propositions contradictoires de la façon suivante: l'art est de l'art pour autant qu'il est possible que ce qui est de l'art, en même temps, ne 
soit pas de l'art. Il est de l'art quand ses productions appartiennent à un milieu sensible où se brouille la distinction entre ce qui est de l'art et ce qui n'en est pas. En bref, le «moyen » est toujours moyen d'autre chose que de sa fin propre. Il est aussi le moyen de participer à la configuration d'un milieu spécifique. La tension entre le médium comme moyen neutre et le médium comme substance propre, entre le médium comme instrument de réalisation d'une idée de l'art et le médium comme ce qui résiste à l'idée et à l'art se résout dans un troisième terme, une troisième idée, le médium comme milieu: le milieu dans lequel les performances d'un dispositif artistique déterminé viennent s'inscrire, mais aussi le milieu que ces performances contribuent elles-mêmes à configurer. Suspendre l'art à la loi du médium, c'est en fait postuler le recouvrement de ces deux milieux. C'est postuler une loi d'adéquation des performances artistiques «fidèles à leur médium » avec un nouveau milieu d'expérience, un nouveau monde technique qui est à la fois un nouveau monde sensible et un nouveau monde social.

3 Au sein de cette vision, la photographie a un rôle privilégié. L'appareil photographique, c'est, d'un côté, le pur instrument, l'automate au service de toute volonté, et en particulier au service de l'art en tant qu'il est l'accomplissement d'une volonté de faire de l'art. Mais c'est aussi l'instrument qui exécute par lui-même la tâche de représentation qui était celle de l'art et délivre ainsi celui qui l'emploie du souci de faire de l'art et de la prétention d'être artiste. Il est la technique de la mimesis: pas seulement, ce qu'on invoque toujours, la technique qui libère l'art de la mimesis, mais aussi la technique qui libère la mimesis de l'art, qui permet aux choses de se donner à voir, délivrées des codes de la représentation, des rapports codés entre formes visibles et production d'effets de signification. Cette libération opérée par la machine photographique ou cinématographique- donnant accès à une vérité ou un inconscient du visible a été célébrée par Walter Benjamin aussi bien que par Jean Epstein. Si la photographie, qui nous occupe ici, est par excellence le médium donnant accès à un milieu sensible inédit, l'artiste photographe « fidèle à son médium » est alors celui qui capte ce milieu sensible nouveau, qui inscrit les performances de son appareil dans sa configuration. L'appareil est l'artiste véridique, disait encore Jean Epstein. Mais il y a deux manières d'entendre le rôle de cet artiste véridique, d'entendre le rapport entre sa puissance artistique et sa véridicité. D'un côté l'appareil est artiste, parce qu'il produit une écriture, plus précisément parce que, en lui, c'est une puissance impersonnelle, la lumière, qui écrit. Le milieu sensible nouveau qu'il configure est alors celui où lumière et mouvement constituent une nouvelle écriture. Mais, de l'autre côté, il est artiste véridique parce qu'il n'écrit rien, parce qu'il livre seulement un document, une information, tels que les machines les fournissent aux hommes qui travaillent sur les machines et sont instrumentés par elles, aux hommes qui doivent apprendre d'elles une nouvelle manière d'être mais aussi les domestiquer à leur usage.

La première idée pourrait être illustrée par une exposition qui, en 2005, marqua l'installation du Centre National de la Photographie dans son lieu nouveau du Jeu de Paume. L'exposition s'intitulait Éblouissement. Dans une même salle on pouvait y voir : des photographies cliniques des malades de Charcot, une image du Cabinet du Docteur Caligari, des solarisations de Man Ray, une surimpression de Maurice Tabard, un photogramme de Raoul Haussmann, des photographies de Brassaï, une " décomposition » et une " moire » d'Eric Rondepierre et des photographies de la Danse serpentine de Loïe Fuller. Rien que des photographies donc, mais des photographies de nature et de statut très différents: des photographies avec ou sans caméra, des photographies documentaires et des photographies artistiques, des photographies 
simples ou travaillées, et éventuellement extraites d'autres supports. Une certaine idée du médium photographique unifiait ce rassemblement apparemment hétéroclite: toutes les photographies rassemblées là témoignaient de la découverte d'un autre monde sensible : le monde du mouvement capté et de la lumière qui s'écrit, un monde découvert par les machines à l'intérieur du monde de l'expérience ordinaire: un intérieur du sensible, mais aussi un nouveau monde vécu, celui du mouvement et de l'électricité : un monde où il $\mathrm{y}$ a continuité entre la lumière des réverbères et l'éclair de l'appareil de Brassaï découvrant sur les murs les hiéroglyphes du rêve. C'est cette identité d'une nouvelle physis et d'un nouveau monde vécu qui met ensemble la danse lumineuse de Loïe Fuller, les féeries nocturnes de Brassaï et les rayogrammes ou solarisations de Man Ray. Le médium photographique, c'est le moyen d'enregistrer mais aussi de contribuer à former ce nouveau monde des machines : un monde de la technique, mais où toute les techniques s'indifférencient : un calligramme d'Apollinaire ou une toile de Boccioni auraient été tout aussi bien à leur place ici. L'idée du médium déborde en effet clairement l'idée de l'appareil. Et il faudrait sans doute, plutôt que de médium, parler ici de médialité, en entendant par là le rapport entre trois choses : une idée du médium, une idée de l'art et une idée du sensorium au sein duquel ce dispositif technique accomplit les performances de l'art. La médialité ici envisagée implique l'unité immédiate entre la puissance d'un organon et celle d'un sensorium. La photographie- y compris dans ses formes sans appareil - et le cinéma sont les arts de ce nouveau monde sensible où lumière et mouvement sont directement expérimentés et expérimentateurs en même temps: un monde des intensités et des vitesses où la matière se spiritualise en énergie lumineuse et motrice et où la pensée et le rêve ont la même consistance que la matière instrumentée. Le médium comme milieu absorbe en fait le médium comme instrument. L'appareil - photographique ou autre - crée un nouveau monde sensible pour autant qu'il nie sa propre spécificité dans un monde de l'expérimentation généralisée. C'est cette indifférenciation, cette dé-technicisation de la technique qui est l'opération fondamentale en jeu sous divers vocables d'écoles: simultanéisme, futurisme, surréalisme ou autres.

5 À cette vision s'oppose clairement une autre manière de penser à la fois le rôle de la machine artiste et le rapport entre le médium technique et le milieu sensible. Dans cette perspective, ce que l'instrument technique produit, ce ne sont pas les épiphanies d'un nouveau monde sensible, mais des documents, des traces, des indices qui doivent être regardés, lus, interprétés, utilisés. C'est en particulier la position de Benjamin dans sa Petite Histoire de la photographie et dans L'œeuvre d'art à l'époque de la reproductibilité technique. Une tonne de commentaires a été déversée sur cette malheureuse reproductibilité dont, paradoxalement, Benjamin parle fort peu, une autre tonne sur l' aura qui lui fait pendant. On en a oublié que le cœur de la démonstration ne porte pas sur les effets de la reproduction en série ; elle porte bien plutôt sur la décomposition de l'unité, sur sa fragmentation en une série d'opérations qui ont valeur de tests, d'enquêtes sur la réalité. L'important, pour Benjamin, n'est pas que les photographies d'Atget ou de Sander soient reproductibles à l'infini. Il est d'abord que ce sont des produits de l'âge des machines, de l'âge de l'existence en masse et de l'homme de masse et que ces produits sont aussi des moyens d'exercer les contemporains à déchiffrer ce nouveau monde vécu et à s'y orienter. Ici encore, mais dans une autre perspective, le privilège de la technique est lié à une indifférenciation des techniques: le cinéma est d'abord une série de "tests " sur notre monde; les photos d'Atget sont des indices à interpréter ; les recueils de Sander sont des « cahiers d'exercice » pour apprendre aux 
combattants de la lutte sociale à identifier partenaires et adversaires. Les produits de la technique reproductrice sont ainsi les moyens d'une nouvelle éducation sensible, les instruments de formation d'une nouvelle classe d'experts en art, en l'art d'interpréter signes et documents. C'est pourquoi Benjamin déclare l'insuffisance de la photographie et la nécessité de la légende qui l'interprète. C'est aussi pourquoi le statut des arts de la reproduction mécanique n'est pas différent de celui du théâtre épique de Brecht, ce théâtre qui est à la fois une école et un parlement, où il faut s'instruire en jouant, en regardant, en discutant. Il faut que les hommes qui travaillent sur les machines de production de masse et vivent au milieu de leurs produits apprennent à se saisir des moyens et des produits de la technique de masse. Il s'agit de former au sein de ce sensorium global qui s'appelle l'être de masse le sensorium particulier des hommes des masses capables de lire les signes sociaux et de s'approprier la production de masse.

6 J'ai évoqué très rapidement ces deux visions du «milieu » de la photographie, afin de présenter la thèse que je souhaite défendre: l'idée de la spécificité du médium est toujours une idée de la médialité, une manière de nouer trois choses: un dispositif technique, une idée de l'art et la formation d'un milieu sensible spécifique. Ces matériaux et instruments de l'art que l'on invoque au titre de la loi du médium sont toujours plus en effet que des matériaux et instruments. Ils sont dotés en fait d'une fonction esthétique, qui est d'imposer un mode de présentation sensible à la place d'un autre. La pensée du médium est toujours en même temps une pensée de l'art et du sensorium qu'il contribue à former. Ainsi cette surface plane revendiquée par Greenberg est bien plus qu'une négation des illusions de la troisième dimension. Elle proclame la liquidation de ce temps où l'art nouveau s'identifiait à une expérimentation sensible illimitée ; elle propose un autre lien, un lien à distance entre la production des formes de l'art et celle des formes d'un nouveau monde vécu. En ce sens la « loi du médium » est bien moins une rupture qu'une forme particulière prise par la double exigence constitutive du régime esthétique de l'art: exigence d'une autonomie de l'expérience esthétique et exigence que l'art soit toujours en même temps autre chose que de l'art.

7 À partir de là il est possible d'analyser les variantes de la pensée du médium comme formes de transformation de cette double exigence. Je voudrais le faire en considérant deux analyses du médium photographique qui ont marqué la pensée de la photographie dans le dernier quart de siècle et qui sont aussi deux manières de régler les comptes de la photographie avec l'idée d'un nouveau monde commun.

8 La première est illustrée par les thèses de Barthes dans La Chambre claire. Il y présente une idée bien déterminée du médium: une idée de l'identité entre matérialité technique et sensorialité. Celle-ci peut s'expliciter en trois points : premièrement, la matérialité technique de la photographie est la négation de l'art. La photographie n'est pas de l'art, elle n'est pas une habileté de l'esprit ou de la main. Elle ne nous touche pas comme la réalisation d'une performance d'un artiste. Mais, deuxièmement, cette négation de l'art nie aussi l'idée d'une performance propre de la technique. Elle s'inscrit à l'opposé de ce que la «négation de l'art par la technique » signifie habituellement, à savoir la banalisation par la reproduction multiple ou la prosaïsation qui oblige à regarder les productions de la photographie comme de simples documents sur la réalité. La thèse de Barthes fait de l'appareil lui-même un milieu, à travers lequel la singularité d'un corps se projette vers moi, vient m'atteindre, voire me blesser. L'opération photographique est pour lui un transport médiumnique. Il ramène en 
quelque sorte l'idée de l'écriture de lumière et de la révélation d'un nouveau monde sensible en arrière d'elle-même, à l'âge où les spiritistes voyaient dans la photographie un moyen de communiquer avec les esprits. C'est l'avoir-été du corps qui vient lui-même s'imprimer sur la plaque sensible et, de là, nous toucher sans médiation. À cette seconde thèse, évidement datée d'avant l'âge numérique, vient s'articuler une troisième : le milieu de la reproduction est pour Barthes exactement le contraire de ce qu'il était dans les visions avant-gardistes, à savoir un monde commun, un monde de la banalisation des signes et de l'expérimentation collective. Au contraire la technique est absorbée dans une essence du sensible qui est celle de la singularité absolue.

Mais cette singularité se laisse elle-même entendre de deux façons différentes. En un premier sens, être singulier, c'est n'être comparable à rien d'autre, ne pas pouvoir être mis en relation avec une autre chose, ne pas avoir de sens. On dira alors que la photographie attire le regard et provoque l'affect sans raison, ou plutôt à cause même de cette absence de raison. C'est ce que résume la célèbre opposition du studium et du punctum : à la photo qui donne des renseignements et exige une interprétation s'oppose la photo qui attire sans raison vers l'unique qui est sans raison: ainsi, dans la photographie des deux enfants débiles par Lewis Hine, le col Danton du petit garçon et la minuscule poupée au doigt de la fille. Pointer ces deux détails, c'est évidemment évacuer le contexte social et politique de la photo: l'activité d'un photographe qui a systématiquement exploré avec son appareil les lieux de l'exploitation et de la relégation, d'un témoin dont les images appellent l'appréciation du nouvel expert de l'âge des masses appelé par Benjamin. Le col Danton permet de repousser tout cela, de régler silencieusement les comptes avec cette médialité qui liait l'appréciation de la performance photographique à une nouvelle "expertise", à l'expérimentation d'un nouveau monde sensible. Le seul monde sensible dont la photo témoigne est le rapport de l'absolue singularité du spectacle à l'absolue singularité du regard. Il en va de même avec la photographie par Avedon de l'ancien esclave. Ici la procédure est inverse: aucun détail qui détourne d'une lecture politico-sociale. Au contraire le masque du sujet photographié ne dit rien d'autre que la condition d'esclave. Mais l'effet est le même : c'est l'esclavage en personne comme singularité historique qui se donne tout entier dans la singularité d'un seul visage. Décréter l'esclavage présent en personne devant nos yeux, entre nos mains, c'est, en fait, effacer la singularité d'autres photographies qui nous parlent de ce qu'il y a eu entre l'abolition de l'esclavage et notre présent: par exemple cette photographie de John Vachon qui nous montre seulement la pancarte Colored clouée très haut sur le tronc d'un pin, à côté de ce qui est probablement l'objet de sa discrimination, à savoir un robinet. Concentrer sur un visage l'avoir-été de l'esclavage, c'est une manière de régler ses comptes avec la multiplicité des formes d'existence sensible de la discrimination raciale. C'est annuler, au nom d'une transmission intégrale du passé d'un phénomène, cette forme de l'expérience collective qui s'appelait histoire et qui servait naguère de support aux interprétations d'images et aux exercices avec les images. L'opposition du punctum et du studium permet de faire place nette de toute cette tradition d'exercice avec et sur les images. Mais cette suppression ne se fait pas sans reste. Elle vient introduire en retour de singulières distorsions dans l'usage des deux notions.

10 Le meilleur exemple en est donné par la photographie d'un jeune homme dans une cellule. Il est beau, nous dit Barthes, mais cela, c'est le studium. Le punctum, c'est : il va mourir. Le problème est que ce punctum-là n'est pas localisable sur le corps qu'il nous présente. Il n'est aucun événement de l'image, seulement un savoir venu de l'extérieur, 
que nous ne pouvons voir sur la photographie que si nous savons déjà que cette photographie représente Lewis Payne, condamné à mort en 1865 pour tentative de meurtre du secrétaire d'État américain. La prétendue immédiateté du punctum est en fait constituée par la conjonction de deux choses: d'une part, la connaissance de l'histoire du personnage, d'autre part la texture même de la photographie, sa coloration qui nous indique que c'est une photo d'autrefois, la photo de quelqu'un qui, de toute façon, est mort au moment où nous le regardons.

11 La « singularité » prend alors un tout autre sens. Plus que l'être incomparable, ce qui la constitue, c'est le fait d'avoir été là, donc de ne plus y être. La singularité de la photographie est alors celle de l'imago latine, de cette effigie du mort qui devient chez Barthes effigie de la mort. La photographie devient une messagère de l'au-delà. Et cette détermination rabat sur le seul rapport médiumnique ce qui fait l'affect réel de la photographie : dans le cas de Lewis Payne, non pas le savoir qu'il va mourir, mais, au contraire, un non-savoir. À première vue nous ne savons pas qui il est, pourquoi il regarde ainsi. Et, même si nous savons qui est ce jeune homme, nous sommes encore incapables de savoir la pensée animant ce regard qui n'exprime ni peur ni révolte, ni résignation ni repentir. De la même façon nous ignorons ce que pense le photographe, et si c'est à sa demande que le détenu est assis à la frontière de la lumière et de l'ombre, son regard intensément tourné vers lui. L'affect de cette photo vient en définitive de l'impossibilité d'établir aucun rapport déterminé entre la modalité de ce regard et l'imminence de la mort, entre le présent de la manière dont il nous affecte et l'âge de la photographie, entre la singularité et l'anonymat. L'« avoir-été » se décompose en fait en une pluralité de relations dont le rapport indéfini fait pour nous la qualité esthétique de la photographie. Or Barthes rabat cette pluralité sur la seule image de la mort. La mort devient le nom de l'Unique qui est la puissance médiumnique de la photographie parce qu'elle est le pur rapport de ce qui est à ce qui n'est plus sur lequel vient s'écraser cette dimension de l'expérience sensible collective qu'on appelait l'histoire.

12 C'est à une autre forme de réduction que conduit la seconde manière de comprendre la loi du médium que j'évoquais plus haut. À priori, pourtant, celle-ci se situe à l'antipode de la vision de Barthes. Elle affirme en effet que le médium est un instrument, un moyen de reproduction et rien d'autre. Le propre de l'artiste qui l'utilise est précisément de le traiter comme tel, d'utiliser ses ressources comme appareil sans prétendre en faire un milieu ou un sensorium. Cette thèse définit une idée de la photographie que peut résumer le titre d'une exposition et de la publication dirigée par Jean-François Chevrier et James Lingwood qui l'accompagnait: Une autre objectivité. Leur texte donnait comme spécificité au médium photographique sa "pauvreté ontologique ", son absence de consistance ontologique forte, propre à faire du médium un milieu. L'appareil photographique, dans cette optique, est un moyen de fournir une information objective et reproductible sur ce qui est en face de l'objectif. Cette essence du médium condamne deux usages de la photo: l'usage virtuose fondé sur «la disponibilité subjective et l'égalité visuelle", qui associe les capacités de réponse immédiate de l'appareil avec la capacité de saisir l'événement visuel marquant la maitrise de l'artiste ; l'usage émotionnel qui met l'affect à la place de l'information comme dans la " photographie humaniste».

13 Ce double interdit attribué au médium montre assez que cette idée de l'objectivité du médium est elle-même une idée de l'art, une manière de définir une adéquation entre 
l'essence de la photographie et celle de la "modernité » artistique. Le problème est seulement qu'il n'y a pas une seule définition de la modernité. Et l'idée de l'objectivité photographique oscille entre deux idées de sa spécificité qui sont aussi deux idées de la modernité.

D'un côté, la spécificité du médium est assimilée à sa reproductibilité. Être fidèle au médium, c'est alors être fidèle à son essence multiplicatrice. Mais il n'est guère facile de discerner la qualité spécifique qu'une image tient du fait d'être reproductible. Cela l'est d'autant moins que l'existence même de la photographie rend toute image multipliable à l'infini et fait qu'elle nous parvient massivement sous la forme de ses copies. De ce point de vue, il en va du portrait du jeune noble anglais peint par Holbein comme de l'apprentie italienne photographiée par Paul Strand. Aussi, chez Chevrier et Lingwood, cette essence multiplicatrice est-elle déplacée de l'idée de la multiplication de l'un à celle de l'unité multiple. La reproductibilité devient alors sérialité. D'où l'exemplarité conférée aux travaux des Becher. Ceux-ci font des séries, comme August Sander. Mais le problème est que les séries de Sander étaient des typologies. La valeur que leur accordait Benjamin était celle d'une formation du sens physionomiste. Ces séries de types sociaux allemands étaient des moyens d'identification et de lutte, permettant aux combattants de savoir qui ils avaient en face d'eux et de s'habituer à la réciproque. Il n'y a évidemment nul service de ce genre à attendre des séries de châteaux d'eau ou de sites industriels désaffectés des Becher. Celles-ci tomberaient même aisément sous le coup de la critique de Benjamin : la photo des usines ne dit rien sur les rapports sociaux qui s'y manifestent. L'intérêt de la série ne peut donc plus résider en ce qu'elle donne à comprendre des rapports sociaux. Il se ramène à une vertu éthique accordée au multiple en tant que tel, en tant qu'il repousse les prestiges de l'un et de l'aura, de l'instant unique et de la contemplation extatique. Ce principe est purement négatif, comme chez Barthes, même si c'est en un sens exactement inverse. Sa "positivité " artistique lui vient donc d'une seconde manière de penser l'« objectivité » du médium. Celle-ci se résume, chez Chevrier et Lingwood, en une notion, celle de la «formetableau ", exemplifiée par les photographies sur caisson lumineux de Jeff Wall. Mais quel rapport penser entre ces grandes scènes en forme de tableau d'histoire et les rectangles qui font ressembler les vues de hauts-fourneaux des Becher à des planches pédagogiques? Rien peut-être sinon l'idée greenbergienne de la surface qui enferme la performance de l'artiste et lui interdit de sortir de soi, de montrer de l'empathie pour son sujet ou de se prendre pour une forme d'expérimentation sociale. En ce sens les sites industriels désertés des Becher sont une manière de clore le rêve des artistes ingénieurs et constructeurs d'usines du temps de Peter Behrens, tout comme la fascination de Barthes pour le col Danton servait à refouler l'engagement du photographe Lewis Hine aux côtés des damnés de l'usine ou de l'hospice. L'« essence » du médium est ici encore une manière de régler des comptes avec l'époque où le médium était pensé comme l'organon d'un nouveau monde collectif. Simplement ce règlement est plus complexe dans le cas des Becher et des théoriciens de la "photographie objective": le refoulement du rêve constructiviste est aussi l'affirmation d'une fidélité à des valeurs liées à l'univers industriel et au combat ouvrier: la sobriété du regard documentaire qui repousse le pathos humaniste, les principes formels de la frontalité, du cadrage uniforme et de la présentation en série qui lient objectivité scientifique et effacement de la subjectivité de l'artiste.

Reste que ce qui est donné à voir par ce parti-pris objectiviste est fondamentalement une absence: des édifices désaffectés à la place des classes et des types sociaux. 
Photographier l'absence, cela peut s'interpréter de deux façons: ce peut être une manière de montrer l'absentement programmé du monde industriel et ouvrier ; mais c'est tout autant une façon de faire jouer l'affect esthétique du désaffecté qui nous renvoie du côté de l'« avoir été » de Barthes. Cette tension de l'idée « objectiviste » du médium est plus sensible encore dans les séries d'un disciple des Becher, Frank Breuer. Je pense notamment à ses séries de containers, présentées, avec celles des entrepôts et des logos, aux Rencontres photographiques d'Arles en 2005. Ces tirages de format moyen étaient présentés dans le transept d'une ancienne église. De loin on les voyait comme des tableaux abstraits ou des reproductions de sculptures minimalistes. En s'approchant on découvrait que ces rectangles de couleur sur fond blanc étaient des containers empilés sur un grand espace désert. L'impact de la série tenait évidemment à la tension entre ce minimalisme et la signification qu'il recélait. Ces containers devaient être ou avoir été remplis de marchandises débarquées à Anvers ou à Rotterdam, probablement produites en un pays lointain, le Sud-Est asiatique peut-être, par des travailleurs sans visage. Ils étaient en somme remplis de leur absence qui était aussi celle de tout travailleur occupé à les décharger et plus lointainement celle des ouvriers européens remplacés par ces travailleurs lointains.

L'« objectivité » du médium recouvre alors un rapport esthétique déterminé entre opacité et transparence, entre les containers comme présence brute de pures formes colorées et les containers comme représentants du "mystère » de la marchandise, c'est-à-dire de la manière dont elle absorbe le travail humain et cache ses mutations. Elle consiste dans le rapport d'une présence à une absence, dans la double relation d'une forme visible à une signification et à une absence de sens. Il faudrait alors aller jusqu'au bout de l'idée de la "pauvreté ontologique». Elle ne signifie pas que la photographie aurait en partage une "pauvreté en être » déterminant ses possibilités artistiques propres. Elle signifie à l'inverse qu'elle n'est pas sous la loi d'une consistance ontologique propre liée à la spécificité de son dispositif technique, qu'elle se prête donc à accomplir des idées de l'art qui s'inscrivent dans une histoire qui la déborde. On peut le comprendre par analogie avec les analyses d'Eisenstein sur le montage cinématographique. Celles-ci nous montrent comment le montage accomplit ce que d'autres arts ont soit rêvé soit réalisé par leurs moyens propres, par exemple la peinture dans le portrait fait par Serov de l'actrice Yermolova : l'image immobile de la peinture doit traduire dans le portrait de l'artiste posant devant le peintre l'énergie de l'actrice en action .Le peintre y parvient en incluant, grâce aux miroirs et moulures de la pièce, plusieurs cadrages différents pour les parties de son corps, plusieurs " plans » dans un seul . Le cinéma explicite grâce à la technique du montage dans le temps un pouvoir de signification que la peinture approchait par la fragmentation de son espace. C'est un accomplissement du même ordre qu'autorise la photographie en réalisant, à l'inverse un pouvoir d'immobilisation par lequel la littérature s'efforçait de suspendre le mouvement de ses phrases ou une puissance de l'involontaire que la peinture devait recréer par l'artifice de la répartition des touches. La "pauvreté » de la photographie lui permet en bref de réaliser cette inclusion du non-art que la littérature ou la peinture devaient mimer par les moyens de l'art.

17 C'est ce que peut illustrer une photographie qui se situe dans l'intervalle entre l'avoirété de Barthes et l'objectivité de l'école des Becher. Cette photographie de Walker Evans nous représente un détail d'une cuisine dans une ferme de l'Alabama. Elle répond donc d'abord à une fonction documentaire au sein de la grande enquête commissionnée par la Farm Security Administration. Pourtant quelque chose se passe dans la photo qui 
excède le renseignement sur une condition misérable: une cuisine sans buffet ni vaisselier, des couverts en fer blanc tenus dans un râtelier de fortune, une barre de bois de guingois clouée sur un mur de planches disjointes et vermoulues. Ce qui nous retient est une certaine disposition esthétique marquée par le déséquilibre: les parallèles ne sont pas parallèles, les couverts sont assemblés en désordre, les objets sur la planche du haut sont placés d'une manière dissymétrique. Cette dysfonctionnalité compose une dissymétrie harmonieuse dont la cause demeure indécise : est-ce l'effet du hasard, du fait que les choses se trouvaient là ainsi en face de l'objectif ? Est-ce celui du regard du photographe qui a choisi un cadre rapproché sur un détail, transformant ainsi en qualité artistique une disposition toute aléatoire ou simplement fonctionnelle ? Ou bien est-ce le goût esthétique d'un habitant des lieux, faisant de l'art avec les moyens du bord en enfonçant un clou ou en posant un bidon ici plutôt que là ? Il se peut que le photographe ait voulu montrer la misère des fermiers. Il se peut qu'il ait simplement photographié ce qui était devant lui sans intention particulière, et que sa photo bénéficie ainsi de la beauté de l'aléatoire. Il se peut qu'il ait eu plaisir à voir là un tableau minimaliste quasi-abstrait ou qu'il ait voulu, à l'inverse, souligner une certaine beauté du fonctionnel : la sobriété de la planche et du râtelier peut en effet satisfaire une esthétique du design, amoureuse du matériau simple et brut et des arts de faire et de vivre transmis par des générations de simples gens. La qualité esthétique de la photographie tient en somme à un parfait équilibre, une parfaite indécision entre les deux formes de beauté que distinguait Kant : la beauté adhérente de la forme adaptée à sa fonction et la beauté libre de la finalité sans fin.

Devant notre regard, il n'y a dès lors ni simple information objective sur une situation ni blessure du ça a été. La photo ne dit pas si elle est ou non de l'art, si elle représente la pauvreté ou un jeu de droites et de diagonales, de poids et de contrepoids, d'ordre et de désordre. Elle ne dit ni ce qu'avait en tête celui ou celle qui a ainsi disposé les planches et les couverts, ni ce qu'a voulu faire le photographe. Ce jeu d'écarts multipliés illustre exemplairement ce que Kant désignait sous le nom d'idée esthétique: "cette représentation de l'imagination qui donne beaucoup à penser sans qu'aucune idée déterminée, c'est-à-dire aucun concept, puisse lui être adéquate ». L'idée esthétique, c'est l'idée indéterminée qui raccorde les deux processus que la destruction de l'ordre mimétique a laissés disjoints : la production intentionnelle de l'art qui poursuit une fin et l'expérience sensible de la beauté comme finalité sans fin. La photographie est exemplairement un art des idées esthétiques parce qu'elle est exemplairement un art capable de permettre au non-art d'accomplir l'art en le dépossédant.

Je ne veux pas tirer de ces réflexions rapides des conclusions générales propres à valider ou à invalider la thèse qui fait l'argument de cette rencontre: celle d'un "tournant technique» de l'esthétique. Mais je pense qu'on peut en tirer quelques questions propres à éclaircir ce qui est en jeu dans cette question. Ce qui est en jeu, c'est d'abord bien sûr de savoir ce qu'on appelle technique. Sous ce nom on désigne en effet cinq choses au moins. C'est d'abord une capacité de produire certaines opérations ; c'est, en second lieu, le modèle général de rationalité en termes de moyens et de fins; c'est encore la capacité d'un appareil de se substituer aux opérations des gestes humains ; c'est ensuite la forme d'extension de cette substitution, la manière dont elle vient s'emparer des opérations de la main, du regard, du cerveau ; C'est enfin le monde vécu corrélatif à l'ensemble de ces substitutions. Parler de technique, assigner à la technique tel ou tel effet, moment ou tournant, c'est en fait opérer un certain nombre de déplacements entre ces niveaux qu'on ramène trop facilement à quelque 
essence ou projet global comme la « mise à disposition des étants » de Heidegger. Ce qui est en jeu, c'est aussi la manière dont on pense les technicités de l'art, le rapport de ce que nous pratiquons, percevons et pensons comme de l'art avec l'effectuation de certains savoirs au profit de certaines fins. On peut entendre par "tournant technique » de l'art et de l'esthétique la multiplication des appareils qui exécutent par eux-mêmes l'œuvre de la main, du regard ou du cerveau artiste. Mais il y a trois manières de penser cette multiplication et son effet sur l'art.

On peut d'abord la penser comme multiplication des instruments à disposition, permettant à l'art d'être libre de ses fins, seul avec ses matériaux et instruments. C'est la thèse dite moderniste (non sans abus, car il y a plusieurs modernismes et celui-ci est plutôt un modernisme du lendemain ). La thèse bute sur la vieille objection hégélienne : celui qui est seul avec ses matériaux et ses instruments n'a pas de raison de faire ceci plutôt que cela. La réponse à l'objection, fournie exemplairement par Adorno, oblige à donner au matériau une volonté propre, c'est-à-dire en définitive à remettre l'autonomie de l'art à un destin technique, en supposant un impetus immanent à cette matérialité qui récuse le simple modèle instrumental.

21 La deuxième manière pense le tournant technique de l'art comme la prise de ses opérations dans la puissance d'un monde sensible nouveau - le monde des machines, de l'énergie, de l'électricité, plus tard de l'information et de la communication. La technique apparait alors comme le processus global qui dissout la spécificité de l'art. Elle annule en effet sa prétention d'être plus qu'une technique, plus qu'une modification des énergies du monde ou une pratique instrumentale d'enregistrement des choses et de mobilisation des énergies. Elle réalise l'art en le supprimant, en faisant de ses formes des formes de la vie. C'est la vision qui mérite plus proprement la qualification moderniste. Mais, nous l'avons vu, ce modernisme connaît deux grandes versions. La première absorbe les manifestations de l'art dans le vaste ensemble des intensités, vitesses et dynamismes qui composent le nouveau monde sensible. La seconde fait de ces manifestations des documents sur ce monde ou des instruments pour l'interpréter et s'y orienter. C'est cette version qui est aujourd'hui rabattue sur la platitude de la démystification qui renvoie les différences illusoires de l'art à la généralité des formes de la technique et de la marchandise.

Mais il y a encore une troisième manière de penser l'effet de la multiplication des appareils. On y voit moins la multiplication des instruments au service des fins des arts que celle des moyens par lesquels les techniques différentes s'indifférencient et se prêtent à réaliser une idée de l'art qui est celle de la déspécification. Cette indifférenciation pourtant ne signifie pas la suppression de l'art dans un monde d'énergie collective réalisant le telos de la technique. Elle implique plutôt une neutralisation qui autorise des transferts entre les fins, les moyens et les matériaux des différents arts, la création d'un milieu propre d'expérience qui n'est déterminé ni par les fins de l'art ni par celles de la technique mais s'organise selon les recoupements nouveaux entre arts et techniques, comme entre l'art et ce qui n'est pas l'art. La multiplication des appareils contribue alors à créer des zones de neutralisation où les techniques s'indifférencient et échangent leurs effets, où leurs produits se présentent à une multiplicité de regards et de lectures, des zones de transfert entre les modes d'approche des objets, de fonctionnement des images et d'attribution des significations. On peut ainsi penser une médialité qui échappe aux téléologies de la fin impérieuse ou 
du moyen dévorant la fin, qui ne reconduise ni une idée de la souveraineté de l'art, ni une idée de la dissolution de l'art dans le monde technique.

\section{AUTEUR}

\section{JACQUES RANCIÈRE}

Philosophe français, professeur émérite à l'université de Paris VIII (Saint-Denis) 